\title{
ANCA-Associated Vasculitis Following Johnson and Johnson COVID-19 Vaccine
}

\author{
Rukesh Yadav ${ }^{1}$, SANGAM SHAH ${ }^{2}$, and Santosh Chhetri ${ }^{3}$ \\ ${ }^{1}$ Maharajgunj Medical Campus, Institute of Medicine, Tribhuvan University, Maharajgunj, \\ 44600, Nepal \\ ${ }^{2}$ Maharajgunj Medical Campus, Institute of Medicine \\ ${ }^{3}$ Tribhuvan University Institute of Medicine
}

November 1, 2021

\begin{abstract}
Antineutrophil cytoplasmic autoantibodies associated vasculitis(AAV) is characterized by antibodies against antigens in cytoplasmic granules of neutrophils and predominantly affects small vessels. AAV after COVID-19 mRNA vaccination has been reported. We report rare case of AAV in a patient who presented with rapidly progressive glomerulonephritis(RPGN) after Johnson \& Johnson vaccine administration.
\end{abstract}

\section{Introduction}

As of early September 2021, there are 24 COVID-19 vaccines approved for SARS-CoV-2 prevention, while there are 140 other COVID-19 vaccine candidates in different phases of clinical trials. Approved vaccines have been granted emergency use authorization for the prevention of COVID-19, the ongoing global health pandemic. Although the short term and the common adverse effects of COVID-19 vaccines are well documented, the rare adverse effects are also more likely to be reported due to widespread mass immunization programs. The long-term sequelae of COVID-19 vaccines are yet to be witnessed in the future.

Vasculitis is the disorder characterized by the presence of mononuclear cells in vessel walls of different organs with reactive damage to mural structures. Antineutrophil cytoplasmic autoantibodies (ANCA) associated vasculitis $(\mathrm{AAV})$ is characterized by antibodies against antigens in cytoplasmic granules of neutrophils and predominantly affects small vessels. AAV is associated with ANCA specific for myeloperoxidase (MPOANCA) or proteinase 3 (PR3-ANCA). AAV can lead to renal failure by involving glomeruli. Although AAV after influenza vaccination has been previously reported, there are a very few case reports of AAV following COVID-19 vaccination (mRNA vaccines)(1,2). Here, we report a rare case of AAV in a patient after Johnson \& Johnson COVID-19 vaccine administration.

\section{Case presentation}

A 52-year-old female presented to our center with complaints of fever, joint pain, and weakness of all limbs. She developed symptoms 12 days after getting vaccinated with Johnson and Johnson COVID-19 vaccine in late July 2021. The fever occurred for 10 days, intermittent type, and associated with chills and rigor. The joint pain was for 3 days which was acute in onset involving small joints of hands and feet. The joints were tender making her bed ridden. Concurrently she also developed weakness of all four limbs with relatively more weakness in lower limb than upper limbs. She had more proximal weakness than distal weakness. She had hypertension for 12 years for which she was on medication with amlodipine and enalapril. She had history of hysterectomy for uterine malignancy nine years back. She did not suffer from COVID-19 prior to 
presentation to our center. She is an ex-smoker and consumes alcohol occasionally. She had no drug allergy or use of illicit drugs.

On examination she was ill looking. She had no pallor, icterus, lymphadenopathy, clubbing, or cyanosis. Her blood pressure was $130 / 70 \mathrm{~mm}$ of $\mathrm{Hg}$, pulse rate of 84 beats per minute, respiratory rate of 24 breaths/min, and oxygen saturation of $95 \%$ in room air. Systemic examinations were grossly normal.

Peripheral blood smear revealed normocytic normochromic anemia with neutrophilic cytosis. Serologic evaluation was notable for increased C-reactive protein, decreased C3 complement level, normal C4 level, positivity for p-ANCA and c-ANCA (3+). Stool occult blood test was negative. Serology for HbsAg, HIV 1 and 2 antibody, anti-HCV antibody, antinuclear antibody (ANA), leptosira, salmonella, dengue, and scrub typhus were non-reactive. Renal ultrasound showed tiny non-obstructive calculus in both kidneys. The laboratory values are shown in table 1 .

Table 1: Laboratory parameters of the patient

\begin{tabular}{ll}
\hline Laboratory parameters & Values \\
\hline Hemoglobin & $9.5 \mathrm{gm} \%$ \\
Packed cell volume & $28.4 \%$ \\
Total red blood cell count & $330000 \mathrm{cells} / \mathrm{mm}^{3}$ \\
Mean corpuscular volume & $86 \mathrm{f}$ \\
Mean corpuscular hemoglobin & $28.79 \mathrm{pg}$ \\
Mean corpuscular hemoglobin concentration & $33.45 \%$ \\
Total leukocyte count (TLC) & $21000 \mathrm{cells} / \mathrm{mm}^{3}$ \\
Neutrophils & $92 \%$ \\
Lymphocytes & $08 \%$ \\
Monocytes & $0 \%$ \\
Eosinophil & $0 \%$ \\
Basophil & $0 \%$ \\
Platelets & $223000 \mathrm{cells} / \mathrm{mm}^{3}$ \\
Ferritin & $2 \mathrm{ng} / \mathrm{ml}$ \\
Total iron binding capacity & $208 \mathrm{mg} / \mathrm{dl}$ \\
Urea & $28.9 \mathrm{mmol} / 1$ \\
Creatinine & $6.13 \mathrm{mg} / \mathrm{dl}$ \\
Uric acid & $2.4 \mathrm{mg} / \mathrm{dl}$ \\
Sodium & $131 \mathrm{mEq} / 1$ \\
Calcium & $1.7 \mathrm{mmol} / 1$ \\
Potassium & $5.2 \mathrm{mEq} / \mathrm{l}$ \\
Procalcitonin & $0.47 \mathrm{ng} / \mathrm{ml}$ \\
Total protein & $63 \mathrm{gm} / \mathrm{l}$ \\
Albumin & $40 \mathrm{gm} / \mathrm{l}$ \\
Total bilirubin & $0.8 \mathrm{mg} / \mathrm{dl}$ \\
Indirect bilirubin & $0.1 \mathrm{mg} / \mathrm{dl}$ \\
SGPT & $12 \mathrm{U} / \mathrm{L}$ \\
SGOT & $7 \mathrm{U} / \mathrm{L}$ \\
Alkaline phosphatase (ALP) & $66 \mathrm{U} / \mathrm{L}$ \\
Bleeding time & $2 \mathrm{~seconds}$ \\
Prothrombin time & $12.4 \mathrm{~seconds}$ \\
International normalized ratio & 0.92 \\
\hline &
\end{tabular}

Urinalysis revealed 12 red blood cells (RBCs) per high-power field, 4 white blood cells (WBCs) per highpower field, and $0.631 \mathrm{gm} /$ day protein with albumin $(2+)$. A serum creatinine level of $0.8 \mathrm{mg} / \mathrm{dl}$ was reported 
on routine laboratory evaluation few months ago. Echocardiography revealed normal left ventricular ejection fraction $(60 \%)$, mild concentric left ventricular hypertrophy and grade I left ventricular diastolic dysfunction.

Following this she was started on antibiotics, NSAIDS, and methylprednisolone. As the serum creatinine level did not improve, she was referred for nephrology consultation. She was planned for kidney biopsy and was started on cyclophosphamide. Blood transfusion was done. Renal biopsy revealed necrotizing and crescentic glomerulonephritis with insignificant glomerular immune complex deposit suggesting ANCA associated glomerulonephritis in the view of history of ANCA positivity (Figure 1). She was discharged after 10 days of hospitalization.

\section{Discussion}

Local injection site reactions (swelling, itching, redness, tenderness) and systemic symptoms (fever, headache, fatigue, chills, myalgias, and arthralgias) are the most common adverse effects of all type of COVID-19 vaccines. The rare adverse reactions of COVID-19 vaccines like anaphylaxis, vaccine induced immune thrombocytopenia, thromboembolism (arterial thrombosis, cerebral venous sinus thrombosis), myocarditis, pericarditis, and Guillain-Barre-syndrome $(3,4)$. Minimal change disease is also reported with Pfizer Biotech vaccine $(5,6)$. Despite these adverse effects, COVID-19 vaccines are safe and effective as documented by large number of clinical trials.

Johnson \& Johnson vaccine is an adenovirus vector vaccine which has human adenovirus type $26(\operatorname{Ad} 26)$ vector. The vector expresses SARS-CoV-2 spike (S) antigen without virus propagation which elicits immune response to $\mathrm{S}$ antigen providing protection against COVID-19. Thrombotic complications associated with thrombocytopenia and Guillain-Barre syndrome are the rare reported adverse effect of Johnson \& Johnson COVID-19 vaccine (7). AAV has been not reported previously as the adverse reaction of this vaccine. Here, we report a newly onset renal limited AAV in a 52 year-female after COVID-19 vaccination. She had normal renal function and laboratory parameters prior to vaccination, and this raises the suspicion of potential association between AAV and COVID-19 vaccine.

Genetic factors, infectious agents, some drugs, and environmental factors are thought to initiate the pathogenesis of AAV. There are studies suggesting the potential association between various infections and the occurrence of vasculitis (8). However, whether the infections or vaccinations are the triggering factors for autoimmune conditions like vasculitides still lacks direct evidence. A study pointed out the increased level of ANCA in patients immunized with RNA based influenza and rabies vaccine $(9,10)$. Furthermore, there have been reports of AAV and other autoimmune conditions due to SARS-CoV-2 infections and vaccinations (mRNA COVID-19 vaccines) (11,12). Interestingly, our patient developed AAV following adenovirus vector vaccine in contrast to the reports of AAV and glomerulonephritis following m-RNA COVID-19 vaccines $(1,2)$. The temporal causal association between autoimmune manifestations like AAV and COVID-19 vaccines can be explained by hypothesized mechanisms like molecular mimicry, defective neutrophilic apoptosis, polyclonal activation, and systemic proinflammatory cytokine response. These mechanisms are likely to trigger autoimmune responses in genetically susceptible individuals. Still there are many research going on to fill the research gap on the development of ANCA associated with COVID-19 vaccines.

There are only few case reports of AAV and ANCA glomerulonephritis developing after mRNA COVID-19 vaccination (Pfizer-BioNTech and Moderna) in the literature (1,2). AAV following adenovirus vector vaccine has not been reported previously. We believe that this is the first case report of AAV that occurred following adenovirus vector COVID-19 vaccine administration. Our patient had been immunized with Johnson and Johnson COVID-19 vaccine and developed AAV 10 days after the immunization. There have been reports of AAV after administration of influenza vaccine $(10,13)$. So, as a lesson learnt from the past, we can speculate the neutrophilic immune response to adenovirus vector present in Johnson and Johnson vaccines, to be a potential trigger for development of AAV. Derangement in the function and expression of MPO and PR3 present in neutrophil granules have been known to cause pauci-immune vasculitis. Although this case does not conclusively clarify the causal relationship between adenovirus vector COVID-19 vaccine and AAV, further study in the future will insight the association. 


\section{Conclusion}

Increasing reports of rare adverse effects like AAV following COVID-19 vaccination warrants the further study and evaluation of immune responses induced by those vaccines. Considering the potential severity of COVID-19 and the rarity of the above-mentioned adverse effects, COVID-19 vaccination should not be withheld. It is evident that the overall benefit of vaccination likely outweighs the risk of adverse side effects of COVID-19 vaccines including AAV for most individuals.

\section{Acknowledgement}

None

\section{Funding}

No funding was received for the study

\section{Ethical consent}

Written informed consent was obtained from the patient for publication of case report

\section{Data availability statement}

All the required information is in manuscript itself

\section{Conflict of interest}

None

\section{Authors' contribution}

RY wrote the original manuscript, reviewed, and edited the original manuscript. SS reviewed and edited the original manuscript. RY, SS, and SC reviewed the manuscript and were in charge of case.

\section{References}

1. Shakoor MT, Birkenbach MP, Lynch M. ANCA-Associated Vasculitis Following Pfizer-BioNTech COVID19 Vaccine. Am J Kidney Dis. 2021 Oct;78(4):611.

2. Sekar A, Campbell R, Tabbara J, Rastogi P. ANCA glomerulonephritis after the Moderna COVID-19 vaccination. Kidney Int. 2021 Aug;100(2):473.

3. Shimabukuro TT, Cole M, Su JR. Reports of Anaphylaxis After Receipt of mRNA COVID-19 Vaccines in the US-December 14, 2020-January 18, 2021. JAMA. 2021 Mar;325(11):1101-2.

4. Team CC-19 R, Administration F and D. Allergic Reactions Including Anaphylaxis After Receipt of the First Dose of Moderna COVID-19 Vaccine - United States, December 21, 2020-January 10, 2021. Morb Mortal Wkly Rep. 2021 Jan;70(4):125.

5. Lebedev L, Sapojnikov M, Wechsler A, Varadi-Levi R, Zamir D, Tobar A, et al. Minimal Change Disease Following the Pfizer-BioNTech COVID-19 Vaccine. Am J Kidney Dis. 2021 Jul;78(1):142.

6. D'Agati VD, Kudose S, Bomback AS, Adamidis A, Tartini A. Minimal change disease and acute kidney injury following the Pfizer-BioNTech COVID-19 vaccine. Kidney Int. 2021 Aug;100(2):461.

7. E J, K P, P R, G M, I A. Facial Diplegia: A Rare, Atypical Variant of Guillain-Barré Syndrome and Ad26.COV2.S Vaccine. Cureus [Internet]. 2021 Jul 25 [cited 2021 Oct 4];13(7). Available from: https://pubmed.ncbi.nlm.nih.gov/34447646/

8. Van Timmeren MM, Heeringa P, Kallenberg CGM. Infectious triggers for vasculitis. Curr Opin Rheumatol. 2014;26(4):416-23. 
9. Jeffs LS, Nitschke J, Tervaert JWC, Peh CA, Hurtado PR. Viral RNA in the influenza vaccine may have contributed to the development of ANCA-associated vasculitis in a patient following immunisation. Clin Rheumatol 2015 354. 2015 Sep;35(4):943-51.

10. Watanabe T. Vasculitis Following Influenza Vaccination: A Review of the Literature. Curr Rheumatol Rev. 2017 May;13(3).

11. Hussein A, Khalil K AL, Bawazir YM. Anti-Neutrophilic Cytoplasmic Antibody (ANCA) Vasculitis Presented as Pulmonary Hemorrhage in a Positive COVID-19 Patient: A Case Report. Cureus. 2020 Aug;12(8).

12. Duran TI, Turkmen E, Dilek M, Sayarlioglu H, Arik N. ANCA-associated vasculitis after COVID-19. Rheumatol Int. 2021 Aug;41(8):1.

13. Zafrir Y, Agmon-Levin N, Shoenfeld Y. Editorial: Post-influenza vaccination vasculitides: A possible new entity. J Clin Rheumatol. 2009 Sep;15(6):269-70.

Figure 1 (a) and 1 (b): Renal biopsy showing necrotizing and crescentic glomerulonephritis with insignificant glomerular immune complex deposit suggesting ANCA associated glomerulonephritis.

Table 1: Laboratory parameters of the patient

\section{Hosted file}

ANCA vasculitis figure.docx available at https://authorea.com/users/444090/articles/543902-

anca-associated-vasculitis-following-johnson-and-johnson-covid-19-vaccine 